\title{
LINEAMIENTOS PARA LA POLÍTICA PÚBLICA DE JUVENTUD DEL MUNICIPIO DE NEIVA, COLOMBIA
}

\section{GUIDELINES FOR THE PUBLIC POLICY OF YOUTH IN THE CITY OF NEIVA}

\section{German Darío Hémbuz Falla1}

Beatriz Peralta Duque ${ }^{2}$

\section{Patricia Gutiérrez Prada ${ }^{3}$}

Universidad de Manizales

\section{RESUMEN}

Se trata de una reflexión sobre un proyectode investigación que brinda lineamientos para la construcción de política pública municipal de juventud en Colombia, caso Neiva capital del Departamento del Huila en el Surcolombiano. Y se presenta el resultado de la firma de un 1 Doctor en Ciencias Sociales, Niñez y Juventud del Centro de Estudios Avanzados en Niñez y Juventud Convenio CINDE y Universidad de Manizales. Docente Universidad Surcolombiana. hembuz@usco.edu.co Orcid: 0000-0002-9976-8225

$2 \quad$ Doctora en Ciencias Sociales Niñez y Juventud del Centro de Estudios Avanzados en Niñez y Juventud Convenio CINDE y Universidad de Manizales, Trabajadora Social, Universidad de Caldas. Magíster en Desarrollo Social y Educativo CINDE; Profesora Doctorado en Ciencias Sociales Niñez y Juventud, Universidad de Manizales (CINDE); Profesora Postgrados en Diseño y Creación; Universidad de Caldas. Correo: beatriz.peralta@umanizales.edu.co 0000-0002-7036-4518

3 Candidata a Doctor en Modelado de Políticas y Gestión Pública de la Universidad Jorge Tadeo Lozano, Magister en Políticas Públicas Universidad del Valle y Administradora de Empresas de la Universidad Surcolombiana. Docente de Planta Facultad de Economía y Administración de la Universidad Surcolombiana. Correo: patricia.gutierrez@usco.edu.co 0000-0002-2928-5906 convenio interadministrativo entre el Municipio de Neiva y la Universidad Surcolombiana, que tuvo como fin desarrollar un ejercicio participativo que vinculó a los jóvenes de la ciudad desde su propia identidad con el arte y la cultura, el deporte, el trabajo, la educación secundaria, la educación superior, las áreas rurales, las comunas, la comunidad LGBTI y la comunidad indígena. Mediante una metodología mixta que desde el interés cuantitativo ofreció una encuesta de caracterización sociodemográfica que contribuyó al análisis de información cualitativa producto de la recolección de información en 12 grupos de discusión y 6 cartografías sociales con jóvenes bajo el enfoque diferencial. Se reconoce la importancia de la apropiación de los conceptos de política pública de juventud con los que se insiste, desarrollar un ejercicio con sentido de participación y enfoque generacional; porque los intereses de la juventud cambian con el tiempo. El documento termina con 
los resultados del estudio exponiendo los lineamientos para una política pública de juventud, a partir de la problematización de 12 categorías de análisis y proponiendo 3 ejes estratégicos para la definición de una política pública municipal.

\section{PALABRAS CLAVES}

Políticas Públicas, Política Pública de Juventud, Estado de Políticas, Juventud, Participación.

\section{ABSTRACT}

This is a reflection on a research project that provides guidelines for the construction of municipal public policy of youth in Colombia, a study case of Neiva as the capital of Huila Province in the Surcolombiano. There is an introduction from the bases of the study that through an inter-administrative agreement between the Municipality and the Surcolombiana University allowed to develop a participatory exercise with young people of the city who have their own identity over the art and culture, job, high school, higher education, rural areas, communes, the LGBTI community and the indigenous community. Through a mixed methodology that from the quantitative interest offered a survey of sociodemographic characterization that contributed to the analysis of qualitative information that was collected in 12 groups of discussion and 6 social cartographies with young people under the differential approach. We recognize the importance of the appropriation of the concepts of public policy and youth policy with which insists on developing an exercise with sense of participation and generational approach; because the interests of youth change over time. The document ends with the results of the study that exposes the guidelines for a youth policy, that from the problematic of 12 categories of analysis they propose 3 strategic axes for the definition of a municipal public policy.

\section{KEY WORDS}

Public Policy, Youth Policy, State of policies, Youth, Participation.

\section{INTRODUCCIÓN}

El Municipio de Neiva, perteneciente al Departamento del Huila de acuerdo a la división político administrativa en Colombia, tiene una extensión de $1.553 \mathrm{Km}^{2}$, con un área estimada de 4.594 hectáreas en la zona urbana y 150.706 en la zona rural, que abarcan desde la Cordillera Oriental hasta la cordillera central y que hacen parte del Valle del Río Magdalena (Alcaldía Municipal de Neiva, 2012 - 2015). Por acuerdo $\mathrm{N}^{\circ} .022$ del ocho de junio de 1995 del Consejo Municipal, en la ciudad Neiva se crearon diez (10) comunas, con 316 barrios y ocho (8) corregimientos establecidos por 78 veredas cuyos centros poblados son Guacirco, Fortalecillas, Caguán, Aipecito, Chapinero, San Luis, Vegalarga y Motilón. En su hidrografía está la cuenca alta del río Magdalena, Río Las Ceibas, Río El Oro, Río Fortalecillas, Río Bache y Río Aipe (Alcaldía Municipal de Neiva, 2012 - 2015). Bajo las proyecciones del censo realizado en el año 2005, la población de Neiva se estima en 345.806 habitantes para el año 2017 , de los cuales 91718 tienen entre 14 y 28 años que corresponde al $26,35 \%$ de la población (Departamento Administrativo Nacional de Estadística - DANE).

El Concejo de Neiva mediante acuerdo 016 de 2011 adopta por primera vez una política pública de juventud para el Municipio de Neiva según la atribuciones que le confieren la Constitución Política de Colombia de 1991 y en especial la primera Ley de Juventud declarada en el año de 1997. En la actualidad, el Estado desde el orden nacional ha reconocido que la juventud en Colombia debe considerarse como una de las fuerzas participativas de mayor relevancia para la construcción de futuro del País y por lo tanto a través de su ley 1622 de 2013 reglamentó el 
Estatuto de Ciudadanía Juvenil que garantiza para la población joven:

"el goce efectivo de los derechos reconocidos en el ordenamiento jurídico interno y lo ratificado en los Tratados Internacionales, y la adopción de las políticas públicas necesarias para su realización, protección y sostenibilidad; y para el fortalecimiento de sus capacidades y condiciones de igualdad de acceso que faciliten su participación e incidencia en la vida social, económica, cultural y democrática del país" (Estatuto de Ciudadanía Juvenil, 2013).

Ante las declaraciones emanadas del Estatuto de Ciudadanía Juvenil, la Administración Municipal de Neiva en el año 2015, soportada en el acuerdo 036 del Consejo Municipal (2014) , decidió producir esfuerzos desde la Secretaría de la Mujer, Infancia y Familia que motivaron eficiencia presupuestal lo que podría ser contraproducente para hacer realidad el alcance de los ejes estratégicos definidos en el proyecto de políticas que incluye los lineamientos que fueron objeto de estudio. Sin embargo, las acciones de renovación del Consejo Municipal de Juventud y foros permanentes de discusión sobre la actualización de la política pública, están reactivando los procesos de formalización requeridos para la aprobación de una política pública de juventud (Proyecto de Acuerdo Política Pública de Juventud, 2016).

La construcción de la política pública de juventud vigente en el municipio de Neiva (Política pública de infancia y adolescencia, 2010) está soportada por los criterios de la antigua ley de juventud 375 (1997) y que fue derogada por la ley estatutaria 1622 de 2013 (Estatuto de Ciudadanía Juvenil, 2013).

Durante el proceso de formulación de la política pública de juventud del Municipio de Neiva se abrieron espacios de participación con enfoque diferencial donde los jóvenes plantearon su sentido crítico de apertura a posibilidades y capacidades que delimitaron la construcción efectiva de lineamientos, los que podrían ofrecer o no oportunidades para el mejoramiento de la calidad de vida de la juventud.

El objetivo de esta reflexión es exponer resultados de una investigación de contexto que ofrece lineamientos para el desarrollo de la política pública de juventud del municipio de Neiva y además de otras consideraciones de tipo normativo, económico, político, social y cultural que se han producido para generar una aproximación crítica de la situación de la política pública de juventud y su necesidad de ajuste relacionándola con la realidad de los jóvenes de la ciudad de Neiva. Secretaría de Equidad e Inclusión a partir del año 2017 (Estructura de la Alcaldía del Municipio de Neiva, 2016), que dio lugar a la desaparición de la Dirección de Juventud por razones de 


\section{METODOLOGÍA}

El proceso de revisión teórica responde a un proceso de vigilancia científica de documentos sobre política públicas de juventud en Colombia y Latinoamérica que incluye el análisis de los documentos CONPES para juventud del Departamento Nacional de Planeación de Colombia, los estudios sobre políticas públicas que han medido variables de desarrollo de las políticas de juventud en Latinoamérica desde los estudios de la UNESCO, OIJ, CELAJU y el COMMONWEALTH. Además, incluye reflexiones de expertos en la investigación de políticas públicas de juventud que llevan a comprender las razones por las cuales se construyó el marco de lineamientos de políticas públicas de juventud en el Municipio de Neiva contrastando los procesos de análisis de políticas públicas de juventud con importantes autores reconocidos en las teorías de la juventud y las políticas públicas.

\section{$\begin{array}{llll}3 & 3 & 1\end{array}$ A MANERA DE DISCUSIÓN: DE LOS LINEAMIENTOS COMO ASUNTO DE REFLEXIÓN DEL CONTEXTO PARA LA POLÍTICA DE JUVENTUD EN LA CIUDAD DE NEIVA}

El desarrollo de la discusión planteada gira en torno al estado de una política pública específica, con tal propósito, inicialmente, se acude a referentes teóricos para conocer y comprender los fundamentos conceptuales de política pública iniciando con Roth (2002) que concibe la política pública como:

"un conjunto conformado por uno o varios objetivos colectivos considerados necesarios o deseables y por medios y acciones que son tratados, por lo menos parcialmente, por una institución u organización gubernamental con la finalidad de orientar el comportamiento de actores individuales o colectivos para modificar una situación percibida como insatisfactoria o problemática" (Pág 27).
Por su pluralidad de conceptos otros autores conciben que una política pública se presenta bajo la forma de un programa de acción gubernamental en un sector de la sociedad o un espacio geográfico, con ello se podría decir que ésta es un programa de acción de una autoridad pública (Meny \& Thoening, 1992) frente a
"situaciones consideradas socialmente problemáticas en cualquier sociedad -desarrollada, en vías de desarrollo, tradicional, moderna, etc.-; hay siempre un conjunto de problemas por resolver. Sin embargo, no siempre el estado puede enfrentarlos a todos por múltiples razones: falta de recursos, tiempo, circunstancias, presiones, intereses, etc. El Estado entonces enfrenta -de manera prioritaria- aquellos que socialmente obedecen a una presión mayor o tienen una mayor incidencia, por lo cual se consideran como problemas socialmente considerados" (Salazar Vargas, 2017).

Según Arroyave Alzate (2011) por políticas públicas se entiende el conjunto de herramientas por las cuales el Estado, una vez identificadas las necesidades económicas, políticas, ambientales, sociales y culturales; ejecuta un conjunto de decisiones que reparan diversos problemas en una construcción participativa que vinculan los actores sociales afectados o que simplemente acude a "un esquema tradicional y tecnocrático sustentado en la formulación e implementación de políticas provenientes desde el Estado; procesos verticales y excluyentes.... donde los grupos afectados por la política son entendidos como centro de análisis y legitimación del proceso" (Arroyave Alzate, 2011, págs. 9697), sin tener en cuenta los actores sociales durante el proceso de toma de decisiones.

Para Alzate (2011), el concepto de políticas públicas se ha desdibujado puesto que los grupos y equipos académicos junto con los funcionarios del Estado no han desarrollado la capacidad de crear un consenso para definir 
qué son, qué hacen y cómo funcionan. Así mismo, los estudios y procesos de ejecución de la política pública carecen de rigor académico y técnico, a razón del corto tiempo durante el que se han aplicado sus orientaciones y el alto nivel de empirismo en su ejecución. No obstante, los procesos de participación en las políticas públicas enfocados en su construcción están dando lugar a la emergencia de axiomas en un entorno local o sectorial.

A todo esto, se debe delimitar o realizar una aproximación teórica sobre lo que se considera como política pública. Para André-Noel Roth Deubel (2002), "existe la política pública siempre y cuando las instituciones estatales asuman total o parcialmente la tarea de alcanzar objetivos estimados como deseables o necesarios, por medio de un proceso destinado a cambiar un estado de cosas percibido como problemático" (p. 27). Asimismo, Alejo Vargas (1998) establece que la política pública es "el conjunto de iniciativas, decisiones y acciones del régimen político frente a situaciones socialmente problemáticas" (p. 169). Por otra parte, Jorge Iván Cuervo (2007) señala al referirse a las políticas públicas que son "el flujo de decisiones en torno de un problema que ha sido considerado público y ha ingresado en la agenda del Estado" (p. 79).

En este orden de ideas, una política pública no es una medida separada del conjunto de decisiones que desde una posición integran a una o más instituciones del Estado; pero que así mismo, llevan a la materialización de una toma de decisiones convertida en acciones que tienen como objetivo producir resultados con relación a una situación problemática con respecto a unos actores sociales involucrados (VargasVelásquez, 2017). Por consiguiente, se puede hablar de la existencia de una política pública, cuando en el nivel local o nacional la autoridad pretende, por intermedio de un programa de acción ordenado, hacer ajustes sobre la realidad situacional de los actores sociales en aspectos como el cultural, social o económico, que se consideran como problemas generalmente intervenidos en la lógica política de un sector (Muller, 2002).

Si bien hay que considerar las limitaciones que se producen una vez se materializan las políticas públicas, es decir tan pronto se dispone su implementación, según Arroyave Alzate (2011), lo importante es asumir varios retos para superar las dificultades en torno al ejercicio de creación, formulación, implementación, evaluación, análisis y reformulación o reestructuración de las políticas públicas considerando que no pueden construirse exclusivamente desde una dimensión conceptual que bajo las ideas de empoderamiento, inclusión, cobertura, planeación y compromiso limitan su ejecución a un proyecto, programa, acuerdo, ordenanza, decreto o ley, dada la relevancia de cumplir con la normatividad nacional o los compromisos de campaña. El segundo desafió a superar tiene que ver con pretender calificar la política pública como posible o ejecutada a plenitud, bajo el principio de inclusión, al creer que se llega a la eficiencia una vez se cede el liderazgo y gestión a terceros, en particular a agentes privados que como administradores de los recursos se ven limitados para vincular a toda la comunidad beneficiada de las políticas, perdiéndose la interacción entre comunidades, Gobierno y grupos de interés como esfuerzo colectivo. Por último, está el desafío de la gobernanza para superar la interdependencia de poderes que se accionan por los funcionarios públicos que necesitan de los políticos para mantener su estabilidad laboral, estos últimos a su vez de los empresarios para financiar sus campañas y de los funcionarios para mantener su liderazgo político, y así mismo la demanda de los empresarios hacia los políticos para sostener las leyes a su favor; así, con dicho triángulo de hierro, no habría lugar para la participación y reclamación de derechos de la sociedad civil. 
Considerando los desafíos para la construcción de políticas públicas, en términos de juventud en Colombia podrían equivocadamente alinearse desde su diferentes territorios al cumplimiento de lo establecido en el Estatuto de Ciudadanía Juvenil; o limitarse a las acciones que lideran organizaciones asociativas para la potencialización de sectores productivos como el caso de los caficultores con la Federación Nacional de Cafeteros que preocupada por el relevo generacional en la producción del grano ha contribuido con la organización de Congresos Juveniles de Caficultores y Clubes Juveniles. Y qué decir de las políticas de planeación territorial que no brindan suficiente cobertura en oportunidades de acceso a la tierra a la juventud rural dado lo intereses de los líderes de la nueva ruralidad por la explotación de biocombustibles y proyectos minero energéticos que se priorizan sobre proyectos productivos sostenibles para la juventud. Por tal motivo, es importante plantear las diferentes posturas de autores sobre la $\begin{array}{lllll}3 & 3 & 3\end{array}$. concepción de juventud o del ser joven a fin de reconocer hacia quienes deben dirigirse decisiones y acciones de política pública las que en Colombia por normatividad se limitan para aquellas personas entre los 14 y 28 años de edad:

Entendemos la noción de juventud como una categoría construida a partir de la relación con el tiempo y el espacio, es decir, como categoría enmarcada en el mundo social (Chaves, 2006), podemos analizar las modalidades en que se "produce la juventud" (Criado, 1998) de acuerdo con experiencias y compromisos vitales, sociales e históricos diferentes, que no hacen sino mostrar los límites que presenta toda clasificación cuyo centro sea solo la edad biológica o una concepción homogenizante de la juventud (Vommaro P. A., 2013, pág. 9).
La definición de juventud es considerada como el "tiempo de espera" hacia la etapa de adultez, que lleva al entusiasmo de la responsabilidad productiva, conyugal, doméstica y parental, y que ha pasado ya a ser una "teoría clásica" que ya no está relacionada con la realidad actual (Souto Kustrín, 2007). Por tal razón se puede decir que es el paso de la etapa hacia la libertad y del encuentro de sí mismo como ser y esto lo lleva al amplio espectro de lo que es la juventud.

Dentro de la explicación dada también se podrán mencionar características físicas y psicológicas de la pubertad para desarrollar una noción biológica de juventud asociada a la adolescencia, comprendida entre los 14 y los 24 años aproximadamente (Dávila León, 2004) (Florenzano Urzúa, 1997), algunos autores relacionan la condición de juventud como un período de resistencia y desordenes emocionales en las que se presenta un desconcierto interno e incertidumbre, que lleva la mayoría de las veces a desajustes en el equilibrio emocional pudiendo ocasionar desórdenes, extravíos y neurosis y que generalmente despierta conductas egoístas, crueles o criminales (Souto Kustrín, 2007); pero hay estudios que muestran la acción colectiva juvenil movilizada por una lucha contra la impunidad y por la solución de conflictos sociales (Aguilar-Forero \& Muñoz, 2015).

Ser joven es una condición que está más allá de la simple edad, la perspectiva o el modo de ver las cosas y de tomar posturas frente a las mismas situaciones, de tal manera ser joven corresponde a una etapa de la vida del ser humano donde la sociedad deja de verlo como niño pero no reconoce su autonomía y estatus como adulto, permitiéndole hacer de manera limitada ciertas acciones dentro de la sociedad con una serie de obligaciones y prohibiciones que se espera puedan ser desarrolladas en su momento oportuno (Aguilar-Forero \& Muñoz, 2015) (Reguillo, 2010) (Feixa, 1998). Así los jóvenes proyectan una ruta para tomar sus 
propias decisiones en términos de conveniencia de sus amigos, ocio, participación en colectivos, educación y empleo para ser sujetos autónomos con un postura clara ante la sociedad (Souto Kustrín, 2007).

La condición juvenil,

cronológicamente, no tiene unos límites de edad precisos ya que, con el paso del tiempo, se ha producido un proceso de ampliación de estos límites -que no dependen sólo de consideraciones psicológicas, sino del desarrollo social, de las posibilidades deindependenciaeconómicaypolítica, de la legislación, o de la percepción de la sociedad, y de los mismos jóvenes $y$ de las organizaciones juveniles-, que continúa en la actualidad. Dentro de este periodo, además, se suele distinguir entre adolescentes $\mathrm{y}$ jóvenes adultos, división que destaca $\begin{array}{lll}3 & 3 & 4\end{array}$ que estos últimos han alcanzado ya ciertas posiciones sociales que no están al alcance de los adolescentes (Souto Kustrín, 2007, pág. 171).

Expertos en políticas públicas de juventud, concluyen que el siglo XXI ha sido enmarcado por una economía y sociedad basada en el conocimiento donde los jóvenes vienen a tener el mayor privilegio para aportarle al desarrollo, pues están considerados como el talento humano que proveerá celeridad a las transformaciones económicas, sociales, tecnológicas, ambientales, culturales y políticas del mundo. Por otro lado, es paradójico el aumento de la exclusión social de los jóvenes para acceder a una educación con calidad, obtener un trabajo digno que garantice su estabilidad y ofrecer participación en un sistema sociopolítico que cuenta con un abanico de oportunidades que se fortalecen desde la institucionalidad (Rodríguez, Políticas publicas de juventud en America Latina: de la construcción de espacios especificos, al desarrollo de una perspectiva generacional, 2003).

Ernesto Rodríguez (2016) presenta un informe del estado de las políticas públicas de juventud en Latinoamérica con las siguientes tensiones e incoherencias:

- Existen políticas sectoriales (salud, empleo, educación, vivienda, entre otros) que incluyen a la juventud como un simple grupo de riesgo mientras se les reconoce como sujetos de derechos y actores estratégicos para el desarrollo.

- Los documentos de política pública priorizan la juventud desde un enfoque diferencial con reconocimiento de su ciudadanía a pesar que los presupuestos llegan para los jóvenes integrados, educación básica y uso positivo del tiempo.

- Se han creado espacios específicos para la juventud pero sin la relevancia de una perspectiva generacional.

- Las instituciones gubernamentales han priorizado pequeños proyectos sectoriales para la juventud que no fomentan la incidencia política en los jóvenes.

- La promoción de Consejos y Clubes de Juventud no está articulada con las grandes movilizaciones juveniles que tan solo han logrado ajustes a la legislación por la juventud pero no producir una incidencia efectiva.

- Las instituciones gubernamentales contratan a jóvenes por su liderazgo y por su preocupación y conocimiento técnico para el fortalecimiento de las políticas públicas.

- Persiste la visión de jóvenes apáticos y desinteresados de lo político mientras emergen colectivos y asociaciones juveniles que no logran incidir en las organizaciones gubernamentales. 
- El discurso de la pluralidad, diversidad y multiplicidad que le es propio a los jóvenes no está presente en los programas y políticas que se refieren a un joven como sujeto homogéneo y unívoco.

- La dinámica poblacional muestra menos niños y más jóvenes pero políticas públicas más dirigidas a los primeros que para los segundos descuidándolos en educación, salud y empleo. Incluso aumenta más la preocupación por políticas para la tercera edad.

- Los programas y servicios para la generación de empleo en los jóvenes promueven la capacitación y adquisición de habilidades básicas para el trabajo pero la orientación laboral es escasa y no logra una efectiva ubicación de los jóvenes.

- La prevención de riesgos y el fomento de una vida saludable en los jóvenes es evidente en $\begin{array}{lllll}3 & 3 & 5\end{array}$ los planes integrales de salud y en la práctica la cobertura se focaliza en la atención de enfermedades específicas.

- Las políticas de acceso a la educación superior en los jóvenes presenta diferencias entre las oportunidades para acceder a universidades públicas con relación a las universidades privadas.

- Los organismos de seguridad del Estado priorizan acciones punitivas para combatir la delincuencia juvenil, por otro lado, las instituciones públicas para la juventud y el desarrollo social previenen la violencia desde una visión de los jóvenes como víctimas y victimarios.

Desde la UNESCO (2010) se ha hecho un seguimiento sobre los alcances de las políticas públicas de juventud en América Latina y en la actualidad los lineamientos acordados para su implementación coinciden con las siguientes prioridades (Rodríguez, 2016):
> Ampliación de la matrícula en educación secundaria y superior principalmente para jóvenes de familia de clase baja y media que demandan procesos metodológicos y curriculares acordes a sus condiciones sociales y no enmarcados sobre los mismos procesos académicos que se brindan para jóvenes de una población de clase alta. Por tal motivo, es importante conocer las tensiones que se originan entre la cultura escolar y la cultura juvenil para que los docentes inviten a un diálogo más franco y efectivo con alumnos y sus familias, además de implementar tecnologías de la información y la comunicación que dinamicen las relaciones docente - estudiante. Se trata también de desarrollar programas académicos que respondan a las demandas del entorno y o no a una oferta de cursos y contenidos que permitan la interacción del joven estudiante en el aula con la realidad del mundo laboral estimulando al empresario generador de empleo juvenil o al joven como emprendedor de sus propias iniciativas de generación de ingresos.

$>\quad$ Promoción de un estilo de vida saludable y pacífico en los jóvenes quienes vienen siendo vinculados con problemas relacionados con infecciones de transmisión sexual, accidentes de tránsito y dinámicas vinculadas a la violencia. Así se ha logrado incorporar los enfoques de seguridad ciudadana, salud pública, de derechos humanos y económicos sobre los fenómenos de las violencias en las que están vinculados directa e indirectamente las personas jóvenes como víctimas o victimarios; generando espacios amigables para la atención de estas problemáticas con personal altamente calificado y campañas de sensibilización que fomentan estilos de vida saludables. Se ha intervenido los problemas de violencia con programas divergentes; algunos con acciones de control social represivos y otros con acciones preventivas diferenciadas entre la población juvenil en general, los que presentan alto nivel de riesgo y los que ya han cometido delitos. 
$>\quad$ Participación juvenil y construcción de ciudadanía para que los jóvenes mediante sistemas de voluntariado lideren programas deportivos, culturales, entre otros, que lamentablemente por falta de interés y presupuesto produce discriminación en la efectividad de las políticas dirigidas a los diferentes grupos sociales de la juventud. Esto ha dado lugar a implementar programas con la participación de los jóvenes bajo el enfoque diferencial apoyados de manera articulada de los Consejos Nacionales y Locales de Juventud.

Con lo anterior, ha surgido la necesidad de potenciar un indicador de desarrollo de políticas sectoriales de juventud que parta de variables como educación media y superior, inserción laboral, salud, prevención de violencias y participación ciudadana (2016), pero dejando abiertas posibilidades para su construcción cuando se consideran otros aspectos relacionados con las problemáticas del territorio, demandas de los movimientos sociales, otras subjetividades e intersubjetividades de la población juvenil.

Las propuestas por un indicador de desarrollo juvenil se empezaron a analizar con los aportes de un estudio mundial propuesto por el Programa de Juventud del Commonwealth financiado por el Institute for Economics and Peace de Londres y reconocido por las Naciones Unidas. Desde el año 2005 se realizan comparaciones entre más de 183 países alrededor del mundo con la medición de 5 dimensiones: educación, empleo, salud, participación política y participación juvenil. Alemania, Dinamarca, Australia, Suiza, Reino Unido, Holanda, Austria, Luxemburgo, Portugal y Japón son los 10 primeros países con mejor índice de desarrollo juvenil. Colombia ocupa el lugar No. 36 en el ranking global, segundo lugar en participación política, 92 en salud, 61 en educación, 100 en empleo y 63 en participación ciudadana (The Commonwealth, 2016).
Para todos los procesos de política pública es necesaria la participación de la sociedad. De manera general la politización juvenil que se despliega en América Latina en la actualidad se evidencia en organizaciones que producen movilizaciones que expresan "posibilidades políticas de establecimiento de relaciones intergeneracionales, a la vez que tienden puentes entre las movilizaciones de los jóvenes y las de otros movimientos y expresiones sociales colectivas más o menos organizadas" (Vommaro P. , 2013). Por ende, los jóvenes, superan los límites entre sectores, etnias, culturas y demás, para transformarse en sujetos de participación que dinamizan diversas luchas sociales más amplias y expresan impugnaciones al sistema dominante que exceden las cuestiones cívicas. Así el concepto de participación
posee un carácter polisémico y está sujeto a una diversidad de variables. La indagación seria según el uso del término en diferentes condiciones, no es lo mismo hablar de participación desde una instancia de poder, que a una instancia subordinada, de resistencia o ciertamente horizontalidad. La participación puede interpretarse como una estrategia de legitimación, además puede entenderse como una aspiración a la inclusión o de ganar visibilidad $\mathrm{o}$ de un orden hegemónico, o también como una forma de potenciación de sujetos sociales (Garcés Montoya \& Acosta V., 2012, pág. 20)

Con respecto a la participación Marx considera que,

La cosa pública es el Estado como asunto real. La deliberación y resolución es la realización del Estado como asunto real. Por tanto no parece sino evidente el que todos los miembros del Estado tengan 
en él su asunto real. Ya el concepto de miembro del Estado supone que cada uno es parte del Estado, que éste les asume como partes suyas. Ahora bien, si son parte del Estado, entonces su misma existencia social será evidentemente su participación real en el mismo. No sólo son parte del Estado, sino que el Estado es su parte. Su parte consciente de algo es tomarse conscientemente una parte suya, tomar conscientemente parte en ello. Un miembro del Estado que careciese de esta consciencia sería un animal... Así que tomar parte en la cosa pública y tomar parte en el Estado es lo mismo (Marx, 2010).

Asumir la participación como participación política da lugar a la construcción de información dirigida hacia "los encargados del gobierno, a los creadores de política pública y a los representantes en general, convirtiéndose en una actividad social enfocada a preservar a la comunidad reflejando esos mismos parámetros sociales" (Vidal Correa, 2014). Una democracia legítima necesita de la participación política pues está en manos de los ciudadanos incidir en las decisiones de los acontecimientos políticos, no es un asunto exclusivo para elegir gobernantes sino también intervenir directa o indirectamente en la formación o construcción de políticas de Gobierno (Biblioteca Virtual del Banco de la República, 2015).

La participación ciudadana y/o política, es un proceso cultural y social. Es cultural, por cuanto la lógica individual propia de la racionalidad occidental debe ser superada en la concreción de propuestas colectivas de grupos y/o comunidades sólidamente construidas, y esto no se logra en poco tiempo en un contexto social mediatizado por el mercado, el consumo y el afianzamiento del capitalismo, privatización del Estado y unificación del modelo económico en el mundo. Es imprescindible: la generación de proyectos pedagógicos sociales y gubernamentales; generación de ambientes políticos propicios para la praxis participativa en la gestión pública local y nacional (Peralta Duque, 2010).

En América Latina los esfuerzos realizados para proponer un índice de desarrollo de políticas sectoriales de juventud desde la Unesco como del Centro Latinoamericano sobre Juventud (CELAJU) en manos de Ernesto Rodríguez (2016) iniciaron con la definición de un Índice de Políticas Integradas de Juventud (no sectoriales) entre 21 países que se organizaron en 3 grupos así: 1) Variables, legales, programáticas e institucionales; 2) Variables vinculadas con generación del conocimiento; y 3) Variables vinculadas con desarrollos de capacidades institucionales. Según los resultados Colombia ocupa el $7^{\circ}$ lugar en políticas integradas de juventud. De la misma forma, se propusieron acuerdos que llevaron a definir el Índice de Desarrollo de Políticas Sectoriales de Juventud objeto de consulta con la institucionalidad de la juventud en los 18 países vinculados para su formalización en América Latina; se recogieron datos agrupados entre el 2005 y el 2013 que midieron 6 categorías: Educación, Inserción Laboral, Educación y Trabajo, Salud, Violencias y Participación Ciudadana. Colombia ocupa el $9^{\circ}$ lugar mientras Brasil, Uruguay, Perú, Costa Rica, Argentina, México, Panamá y Paraguay le anteceden (Rodríguez, Bases para la construcción de un índice de desarrollo de políticas sectoriales de juventud en América Latina, 2016).

La información del Índice de Desarrollo de Políticas Sectoriales para Colombia (Rodríguez, 2016), deja ver que el 95,3\% de los jóvenes entre los 15 y 19 años culminaron su educación básica primaria, $69 \%$ entre los 20 y 24 años culminaron su educación básica secundaria, y $13.2 \%$ de los jóvenes entre los 25 y 29 años terminaron su educación terciaria. La defunciones de jóvenes 
entre 15 y 24 años equivalen al $5.3 \%$ sobre el total de muertes. La tasa de fertilidad de jóvenes entre 15 y 19 años alcanza el 51.7 por mil. Prevalece una tasa del $0,2282 \%$ de $\mathrm{VIH}-\mathrm{SIDA}$ en hombres y $0,1264 \%$ en mujeres de 15 a 24 años. La tasa de participación juvenil insertada laboralmente es del $54.4 \%$, con una tasa de desempleo del $21,9 \%$ y $65.3 \%$ de empleo informal no agrícola juvenil. Los jóvenes que ni estudian ni trabajan suman el $23.2 \%$ de la población juvenil. Así mimo, $59.9 \%$ de los jóvenes de 16 a 25 años participan en elecciones y el $18.7 \%$ entre 16 y 25 años participan en manifestaciones. La tasa de homicidio de jóvenes entre los 15 y 24 años es de 70 por cada 100.000 habitantes. De la población carcelaria $19 \%$ son jóvenes entre 15 y 24 años. Y la tasa de suicidio de jóvenes entre los 15 y 29 años es de 8 por cada 100.000 .

La política pública de juventud en Colombia está considerada como una orientación de actividades del Estado y la sociedad para garantizar los derechos de las y los jóvenes, específicamente relacionada con aquellos derechos que reconocen el ejercicio de su ciudadanía para el desarrollo de sus proyectos de vida (Estatuto de Ciudadanía Juvenil, 2013).

Si bien la historia de la participación juvenil en Colombia y sus bases de lucha por sus derechos han sido de índole político, social, ambiental y cultural, generalmente respondiendo a razones heterogéneas, sobre todo a nivel de 'peso generacional' y por esencia contradiciendo las políticas educativas, sociales, ambientales y culturales. Estos, han llegado a otras dimensiones con relación al antiimperialismo, el anticapitalismo y la solidaridad con organizaciones especialmente conformadas por jóvenes trabajadores, universitarios y estudiantes de secundaria de instituciones públicas y algunas privadas que no hacen parte de las elites nacionales (Archila, 2012). En este sentido se configura en el tiempo una serie de momentos claves que dan auge a las políticas públicas de juventud. En 1968 el Decreto 2743 con el cual se crean el Consejo Nacional y el Instituto Colombiano de la Juventud y el Deporte, Coldeportes, donde en esa época el Estado Colombiano consideraba que los jóvenes sólo se beneficiaban de programas de deporte y recreación (Colombia Joven, 2017). Luego, el Programa de Acción Mundial para los Jóvenes estableció un marco normativo y directrices para la adopción de medidas nacionales y la prestación de apoyo internacional con miras a mejorar la situación de los jóvenes. El 12 de agosto de 1985 las Naciones Unidas inició una celebración anual denominada "El día internacional de la juventud" (ONU, 2017), lo que permitió que por primera vez el tema de juventud tuviera importancia pública. Así se inició una discusión para pensar en los jóvenes como grupo importante, que influye en los diferentes ámbitos del país. Para Hémbuz, Gutíerrez y Caicedo (2015) el nivel de influencia de los jóvenes permitió darle línea a Colombia para implementar programas hacia la juventud, tales como "jóvenes contra las drogas" mediante el decreto 3788 del 86 (Decreto que reglamenta el Estatuto Nacional de Estupefacientes), el cual, les brindaba espacios para prevenir el consumo de sustancias alucinógenas y a su vez el programa de salud promovido por la OMS (1986), dirigido a esta población que no había sido tenida en cuenta. En 1988 el Departamento Nacional de Planeación impulsó lineamientos generales para formular política pública de juventud (DNP, 1988). En 1990 se crea en el país la Consejería Presidencial para la Juventud , la Mujer y la Familia (CONPES, 2014), esta toma vigencia con la publicación del documento CONPES 2626 de "Política Social para los Jóvenes y las Mujeres" (CONPES, 1992), y en el año 1995 se produjo otro documento CONPES 2794 que sirvieron como lineamientos de políticas para ordenar la inversión pública y favorecer las condiciones de vida de la población juvenil (CONPES, 1995). Después de estos documentos, surge la ley 375 de 1997 
denominada Ley de Juventud que establece derechos y obligaciones por parte del estado, allí se construyen los fundamentos legales que forman el Sistema Nacional de Juventud, "entendido como el conjunto de instituciones, organizaciones, entidades y personas que realizan trabajo con y en pro de los jóvenes, cuyo propósito es generar una articulación en la definición y desarrollo de la Política Nacional de Juventud" (Sarmiento Anzola, 2003). Entre ellos los Consejos Municipales de Juventud e instancias estatales como el Viceministerio para la Juventud (1994) y las instancias Distritales, Departamentales y Municipales de juventud.

Pese a la a la promulgación de la ley de juventud en el año 1997, solo a partir del año 2000 mediante el Decreto 089 queda reglamentada la organización y el funcionamiento de los Consejos de Juventud como espacios de participación juvenil y mecanismos de interlocución, veeduría y construcción concertada de las políticas de juventud (Decreto Organización y Funcionamiento de los Consejos de Juventud); en 2001 se inicia la convocatoria al Diálogo para una Política Nacional de Juventud "Presente y futuro de los jóvenes" (Sarmiento Anzola, 2003).

En 2003, de acuerdo con lo establecido en el Plan Nacional de Desarrollo mediante la ley 812 (2003) el Programa Presidencial Colombia Joven tiene como una de sus tareas diseñar y construir, de manera participativa, una Política Pública Nacional de Juventud, que oriente a las entidades y fije prioridades para la intervención del Estado (Sarmiento Anzola, s.f) ${ }^{4}$.

El Plan Nacional de Desarrollo, 2006 - 2010 a través de la Ley 1151 (2007), "reconoce a los y las jóvenes como sujetos activos en la participación política de la vida nacional y como un grupo clave para la generación de una sociedad democrática con un mayor grado de equidad y de armonía" (CONPES, 2014).
Para el año 2013, él Congreso de la Républica de Colombia crea la ley estatutaria 1622, por medio de la cual se expide el Estatuto de Ciudadanía Juvenil, en este se establece una reforma sustancial al establecer un sistema de juventud articulado con los entes territoriales en la toma de decisiones y al obligar a los entes territoriales a crear dependencias con capacidad técnica, financiera y política para la atención de la población juvenil. Además de esto, se crea la Dirección del Sistema Nacional de Juventud "Colombia Joven", dedicada a la promoción de la política pública de juventud a nivel nacional y en los territorios, "fomenta la participación de los jóvenes en los diferentes espacios institucionales; consolida el Sistema de Información y Gestión de Conocimiento SNIGCAJ, articula la oferta pública institucional de juventud y promueve oportunidades socioeconómicas para la juventud" (Colombia Joven , 2017).

Para el año 2011 en el municipio de Neiva, capital del Departamento del Huila, se aprobó la política pública de juventud de Neiva bajo parámetros de la ley 375 de 1997, la cual tenía 4 lineamientos, primero; la promoción y divulgación de los derechos de los jóvenes para su conocimiento como sujetos de derechos. Segundo; la garantía y cumplimiento de los derechos reconocidos para todos los jóvenes del municipio de Neiva, en especial para los jóvenes que se encuentran en situaciones de mayor vulnerabilidad. Tercero; la prevención de la amenaza o vulneración de los derechos de los jóvenes. Cuarto; El impulso y gestión para que los derechos de los jóvenes sean restablecidos cuando han sido violados. (Política pública de infancia y adolescencia, 2010) Cabe resaltar que esa política pública de juventud del municipio de Neiva está soportada por los criterios de la antigua ley de juventud, la cual fue derogada por la ley estatutaria 1622 de 2013. 
Para el año 2015 surge la necesidad de actualizar la política pública de juventud, para llevar a cabo este proceso el Municipio de Neiva firmó el convenio interadministrativo $\mathrm{N}^{\circ} 1319$ con la Universidad Surcolombiana (2015). Dicho documento gracias a una metodología participativa y sin limitarse a los lineamientos de Colombia Joven, expuso las principales problemáticas que abordaban a la juventud en el municipio, trascendiendo el común denominador o las problemáticas siempre analizadas como la drogadicción, prevención de embarazos no deseados y pandillismo. Las problemáticas encontradas fueron analizadas en el marco de distintos grupos diferenciales tales como Jóvenes Trabajadores, Jóvenes de Comunas, Jóvenes Rurales, Jóvenes Víctimas del Conflicto, Jóvenes del Arte y la Cultura, Jóvenes del Deporte y la Recreación, Jóvenes Indígenas, Jóvenes Afros, Jóvenes LGBTI y Jóvenes en Situación de Discapacidad. Se trató de un estudio de tipo exploratorio descriptivo y correlacional, el cual permitió llegar a conocer las situaciones, costumbres y actitudes predominantes a través de la descripción exacta de las actividades, objetos, procesos y personas.

En un primera fase, La realización de 389 encuestas a los jóvenes de los diferentes grupos focales identificados y del resto de la población joven que no participaron en dichos grupos donde se tuvieron en cuenta algunas preguntas de encuestas realizadas por el DANE, como de aquellas que se obtiene en el formato de la caracterización territorial de los y las jóvenes en el Sistema JUACO por lo tanto se trabajaron los siguientes aspectos: Presentación general (edad, estrato, género, condición sexual, cuántos años lleva viviendo en la ciudad), a nivel familiar se examinó el número de personas en el hogar, la relación en el hogar, personas con quien vive actualmente, en el ámbito de educación, si estudian o no, si su institución es pública o privada, el nivel educativo alcanzado también como el de sus padres y a cual aspira actualmente, si se han retirado o aplazado sus estudios, a nivel de salud se preguntó si se encuentra afiliado a la seguridad social en salud, si pertenecen al SISBÉN y el nivel, si han consumido sustancias psicoactivas, el mercado laboral, su participación política, si han sido víctimas del algún hecho del conflicto armado, su perspectiva de la ciudad y la ocupación del tiempo libre. En una segunda fase, se consideró la revisión de fuentes secundarias que permitieran corroborar la información analizada en la etapa de indagación a través de encuestas y entrevistas. $Y$ en la tercera fase, se consolidó la información y el análisis para la elaboración de un informe.

Sobre los resultados Hémbuz, Gutíerrez \& Caicedo (2015), afirman que las principales problemáticas expresadas por la juventud en el Municipio de Neiva, se dan en el marco de los macroproblemas de una sociedad, tales como desempleo, corrupción, acceso a la educación, salud, inseguridad, violencia, escenarios participativos, apatía política por parte de los jóvenes, drogadicción, discriminación, limitada expresión, escenarios de deporte - recreación y cultura, en sí, garantías participativas y de veeduría de los derechos de los jóvenes y la materialización de las políticas (en cuanto a la praxis y desarrollo de las mismas, no se desconoce que existen pero no se desarrollan). Así las condiciones del entorno en el Municipio de Neiva, determinan las necesidades que deben ser satisfechas para cada grupo diferencial de jóvenes reconociendo su identidad social (Berger \& Luckmann, 1999), en términos de las categorías definidas en el cuadro No.1.

El análisis del discurso junto a los resultados que arrojó la caracterización sociodemográfica que se aplicó a los jóvenes del Municipio de Neiva ofreció los siguientes lineamientos para la construcción de políticas públicas: 
- Empleo. Están presentes unas condiciones laborales inadecuadas y de explotación, en donde los tipos de empleo a los cuales tienen acceso los jóvenes son generalmente informales; particularmente por la falta de experiencia laboral.

- Violencias. Inseguridad asociada a los robos, agresiones físicas, discriminación por pertenecer a grupos diversidad sexual o étnica, y actos delictivos propios del conflicto armado son las formas de violencia más comunes.

- $\quad$ Salud. La problemática de salud que más se está vivenciando entre las personas jóvenes es el consumo de drogas tanto en la zona rural como la urbana, sumado a las dificultades de una mala calidad en el servicio.

- $\quad$ Pobreza. Se presenta una vulnerabilidad más marcada en jóvenes de zonas rurales debido a factores de pobreza extrema $y$ violencias especialmente generadas en el marco del conflicto armado especialmente en las zonas rurales.

- $\quad$ Deporte. No hay un apoyo significativo de las instituciones encargadas de promover el deporte, poca continuidad a los proyectos en este campo y no se financian las iniciativas para recuperar los escenarios deportivos.

- Cultura. El ámbito cultural es un escenario sin espacios o son muy escasos; y no todas las expresiones artísticas de los distintos grupos poblacionales de jóvenes tienen acceso a los mismos.

- Institucionalidad y programas. No hay un apoyo de las Alcaldías y Gobernación para programas de jóvenes, además hay un desgaste por parte de los jóvenes pues se sienten utilizados con promesas de campaña electoral.

- $\quad$ Educación. La calidad de la educación secundaria y superior es baja, con dificultades en infraestructura y poco apoyo a los jóvenes para su acceso a becas y programas de bienestar que garanticen su permanencia estudiantil.

- Movimientos I militancias / organizaciones / espacios juveniles. Hay iniciativas de los jóvenes por organizarse para generar espacios de encuentro y participación desde organizaciones políticas, culturales, deportivas, de diversidad sexual entre otras, que demandan más atención y apoyo por parte de las instituciones competentes para los temas de jóvenes y su no estigmatización.

- $\quad$ Ciudad. Ausencia institucional, reflejada en los pocos espacios de encuentro lúdico, recreativo y cultural presentes en la ciudad, los cuales no son óptimos por falta de mantenimiento o por hacinamiento.

- $\quad$ Ser joven. Se refleja en los participantes la condición de ser joven, sentido que se debate entre la pasividad y la proactividad, el ser joven se asocia con ser activo, participativo, propositivo, que exige derechos y se organiza. 


\section{Cuadro No.1.}

\section{Categorías y subcategorías: Fuentes para la formulación de la política pública de juventud del} Municipio de Neiva

\begin{tabular}{|c|c|}
\hline Categorías & Subcategorías \\
\hline Empleo & Condiciones laborales/experiencia laboral/ sentido del trabajo \\
\hline Violencias & Inseguridad/violencias en el marco del conflicto armado \\
\hline Salud & $\begin{array}{l}\text { Consumo de drogas/calidad del servicio de salud/programas de } \\
\text { prevención }\end{array}$ \\
\hline Pobreza & Exclusión / dificultades económicas y educación \\
\hline Deporte & Falta de apoyo institucional; escenarios deportivos/política y deporte \\
\hline Cultura & $\begin{array}{l}\text { Escena cultural sin espacios / arte y su importancia en la vida/arte no } \\
\text { valorada por la institucionalidad }\end{array}$ \\
\hline $\begin{array}{l}\text { Institucionalidad y } \\
\text { programas }\end{array}$ & $\begin{array}{l}\text { Institucionalidad no ofrece apoyo / programas de interés } \\
\text { demandados/jóvenes sin participación en lo institucional }\end{array}$ \\
\hline $\begin{array}{l}\text { Educación básica } \\
\text { secundaria y media }\end{array}$ & $\begin{array}{l}\text { Educación de baja calidad / discriminación en el } \\
\text { contexto escolar / problemática de espacio }\end{array}$ \\
\hline Educación superior & $\begin{array}{l}\text { Calidad educativa / ausencia de beneficios educativos/ dinámicas de } \\
\text { participación }\end{array}$ \\
\hline $\begin{array}{l}\text { Movimientos, } \\
\text { militancias, } \\
\text { organizaciones y } \\
\text { espacios }\end{array}$ & $\begin{array}{l}\text { Falta de espacios lúdico recreativos / privatización de espacios de } \\
\text { encuentro/organización y participación juvenil }\end{array}$ \\
\hline Visión de ciudad. & \\
\hline Ser joven. & \\
\hline
\end{tabular}

Elaboración Propia, 2017

Finalmente, los resultados de la investigación permitieron construir un marco de sugerencias para la actualización de la política pública de Neiva planteado en tres ejes estratégicos (Hémbuz, Gutíerrez, \& Caicedo, 2015), así:

Armonía institucional por la juventud. Plantea acciones interinstitucionales para el empleo, la salud, la educación, la diferenciación sociocultural y las violencias con el propósito de obtener mejores resultados en términos de cobertura y eficiencia. Se prioriza hacer seguimiento sobre los proyectos de vida para los jóvenes; interactuar con todos los sectores sociales; crear comisiones de concertación y decisión activas; y garantizar los derechos de los jóvenes en términos de prevención, promoción y protección. Así mismo, se propuso crear alianzas público-privadas para ofrecer oportunidades para adquirir la primera experiencia laboral y educar para aceptar las diferencias de género y la diversidad.

> Garantías para la participación juvenil. Busca que las instituciones del Estado propendan por promover y difundir deberes, derechos, políticas y programas dirigidos a la juventud como también a la creación de espacios para la participación ciudadana y/o política para la solución de problemas. Así la participación permite dinamizar las acciones del capital social juvenil en las Asambleas y Consejos 
de Juventud y garantizar la autonomía en las plataformas juveniles.

Gestión del conocimiento. Requerida en el territorio para la apertura de espacios de interacción social de los jóvenes en términos de sostenibilidad (medio ambiente, inclusión social, deporte, cultura y recreación). Además activar el conocimiento y talento humano de los jóvenes para la investigación e innovación como solución a las problemáticas de la ciudad, facilitando la planeación con enfoque bottom-up a través de la articulación con el sector educativo y sistemas de información institucionales que permitan la puesta en marcha de los proyectos planteados.

\section{CONCLUSIONES}

Los lineamientos para la política pública de juventud municipal de Neiva consideran dentro de lo planteado:

Una revisión permanente y actualizada al marco teórico de las políticas públicas, que hoy invitan a todos los actores sociales vinculados a participar en el análisis de sus problemáticas y proponer soluciones.

$>\quad$ El marco del alcance de las políticas públicas de juventud invita a establecer un sistema de medición de indicadores de logro de las acciones sociales que permiten el mejoramiento de las condiciones de empleo, salud, educación y participación en los jóvenes; aunque es importante considerar tanto el enfoque de 'peso generacional' y el de territorialidad.

$>$ De igual una política pública de juventud no es posible sin los enfoques de derechos, diferencial, de derechos humanos y de participación como se estable en el Estatuto de Ciudadanía Juvenil.

> Las variables tradicionales para el desarrollo juvenil en el Municipio de Neiva como lo es la educación, el empleo, la salud y la vivienda no garantizan la satisfacción de la calidad de vida de los jóvenes neivanos que además piensan en las problemáticas de la cultura, el deporte, la violencia, la pobreza, la institucionalidad, las organizaciones juveniles, el hábitat y la condición de ser joven.

\section{REFERENCIAS BIBLIOGRÁFICAS}

Acuerdo para la Creación de la Dirección de Juventud de Neiva, 036 (Concejo Municipal de Neiva 30 de Diciembre de 2014).

Aguilar-Forero, N., \& Muñoz, G. (2015). La condición juvenil en Colombia: entre violencia estructural y acción colectiva. Revista Latinoamericana de Ciencias Sociales, Niñez y Juventud, 1021-1035.

Alcaldía Municipal de Neiva. (2012 - 2015). Diagnóstico plan de desarrollo "Unidos para Mejorar". Neiva.

Archila, M. (Mayo de 2012). El movimiento estudiantil en Colombia, una mirada histórica. Observatorio Social de América Latina- OSAL, 31.

Arroyave Alzate, S. (2011). Las políticas públicas en Colombia. Insuficiencias y desafíos. Revista del Departamento de Ciencia Política, Universidad Nacional, sede Medellín, 95 - 111.

Berger, P. L., \& Luckmann, T. (1999). La construcción social de la realidad. Buenos Aires: Doubleday \& Company Inc.

Biblioteca Virtual del Banco de la República. (14 de Junio de 2015). Banco de la República - Colombia - Actividad Cultural. Recuperado el 10 de 2015, de Biblioteca Virtual - Biblioteca Luis Ángel Arango: http://www.banrepcultural. 
org/blaavirtual/ayudadetareas/politica/ participacion_politica

Chaves, M. (2006). Investigaciones sobre juventudes en Argentina: estado del arte en ciencias sociales. Buenos Aires: Instituto de Altos Estudios Sociales.

Colombia Joven . (Marzo de 2017). Colombia Joven: Programa presidencial para el Sistema Nacional. Obtenido de http://wsp.presidencia.gov.co/ ColombiaJoven/elprograma/Paginas/ historia.aspx

CONPES. (23 de Noviembre de 1992). Política social para los jóvenes y las mujeres. Documeno CONPES 2626. Bogotá D.C., Colombia: DNP.

CONPES. (28 de Junio de 1995). Política de juventud. Documento CONPES 2794. Bogotá D.C., Colombia: DNP.

CONPES. (03 de Julio de 2014). Lineamientos para la generación de oportunidades para los jóvenes. Documentos CONPES 173. Bogotá D.C., Colombia: DNP.

Criado, M. (1998). Producir la juventud: Crítica de las sociología de la juventud. Madrid, España: Istmo.

Cuervo, J. (2007). Las políticas públicas entre los modelos teóricos y la practica gubernamental: Una revisión de los presupuestos teóricos de las políitcas públicas en función de su aplicación a ala gestión pública colombiana. En Ensayo sobre políticas públicas. Bogotá D.C.: Universidad Externado de Colombia.

Dávila León, O. (2004). Adolescencia y juventud: De las nociones a los abordajes. Última Década, 83-104.
Decreto Organización y Funcionamiento de los Consejos de Juventud, 89 (Presidencia de la República 7 de Febrero de 2000).

Decreto que reestructura el Ministerio de Educación Nacional, 1953 (Presidencia de la República 8 de Agosto de 1994).

Decreto que reglamenta el Estatuto Nacional de Estupefacientes, 3788 (Presidencia de la República 31 de Diciembre de 1986).

Departamento Administrativo Nacional de Estadística - DANE. (s.f.). DANE. Recuperado el Marzo de 2017, de http:// www.dane.gov.co/

DNP. (1988). Lineamientos generales para la formular una política pública de juventud . Bogotá D.C.: DNP.

Estatuto de Ciudadanía Juvenil, 1622 (Cogreso de la República de Colombia 29 de Abril de 2013).

Estructura de la Alcaldía del Municipio de Neiva, 590 (Alcalde Municipio de Neiva 10 de Octubre de 2016).

Feixa, C. (1998). De jóvenes, bandas y tribus: Antropología de la juventud. Barcelona, España: Editorial Ariel.

Florenzano Urzúa, R. (1997). El adolescente y sus conductas de riesgo. Santiago de Chile: Universidad de Chile.

Garcés Montoya, Á., \& Acosta V., G. L. (2012). Participación política juvenil (primera ed.). Medellin, Antioquia, Colombia: Sello Editorial, Universidad de Medellin.

Hémbuz, G., Gutíerrez, P., \& Caicedo, S. (2015). Actualización de la Política Pública de Juventud. Neiva: Universidad Surcolombiana. 
Ley de Juventud, Ley 375 (Congreso de la República de Colombia 4 de Julio de 1997)

Ley del Plan Nacional de Desarrollo, 812 (Congreso de la República de Colombia 26 de Junio de 2003).

Ley Plan de Desarrollo 2006-2010, 1151 (Congreso de la República de Colombia 24 de Julio de 2007).

Mantilla, L. F. (1999). Algunas Aproximaciones a la participación . Reflexión Política .

Marx, K. (2010). Crítica de la filosofía del Estado de Hegel. Madrid: Biblioteca nueva.

Meny, I., \& Thoening, J. (1992). Las polliticas públicas. (S. Del Carri, Trad.) Barcelona: Editorial Ariel .

Muller, P. (2002). Las Políticas Públicas. (J. F. Jolly, \& C. Salazar Vargas, Trads.)

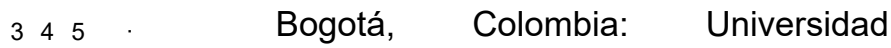
Externado.

OMS. (1986). Cuarto informe de la comisión B. 39 Asamblea Mundial de la Salud (págs. 1-5). Ginebra - Suiza: OMS.

ONU. (10 de Junio de 2017). Día Internacional de la Juventud. Obtenido de http://www. un.org/es/events/youthday/background. shtml

Peralta Duque, B. (2010). La forma en la que se ejerce ciudadanía en en la democracia colombiana: - Socialización política y cultura ciudadana-. Eleuthera, 253-290.

Política pública de infancia y adolescencia, 016 (Concejo de Neiva 22 de Julio de 2010).

Proyecto de Acuerdo Política Pública de Juventud, 043 (Concejo de Neiva 30 de Septiembre de 2016).
Reguillo, R. (2010). Los jóvenes en México. México D.F.: Fondo de Cultura Económica.

Rodríguez, E. (2003). Políticas publicas de juventud en America Latina: de la construcción de espacios especificos, al desarrollo de una perspectiva generacional. Revista Latinoamericana de Ciencias Sociales Niñez y Juventud.

Rodríguez, E. (2016). Bases para la construcción de un índice de desarrollo de políticas sectoriales de juventud en América Latina. Uruguay: Centro Latinomericano sobre Juventud - CELAJU.

Roth, A.-N. (2002). Políticas Públicas, formulación, implementación y evaluación. Bogotá: Ediciones Aurora.

SABUCEDO, J. M., \& SOBRAL, J. (1986). PARTICIPACIÓN POLÍTICA Y CONDUCTA DE VOTO. Papeles del Psicólogo.

Salazar Vargas, C. (2 de Junio de 2017). Carlos Salazar Vargas - Politing: mas allá el marketin político. Obtenido de http://carlosalazarvargas.org/index. php/2017/02/06/definicion-de-politicapublica-de-salazar-csv-en-el-politinguna-opcion/

Sarmiento Anzola, L. (2003). Política Pública de Juventud en Colombia: Logros, dificultades y perspectivas. Bogotá D.C.: Corporación Paisa Joven.

Souto Kustrín, S. (2007). Juventud, teoría e historia: la formación de un sujeto social y de un objeto de análisis. HAOL Numero 13, 192 páginas. 
The Commonwealth. (2016). Global Youth Development Index and Report 2016. London: Commonwealth Secretarial.

UNESCO. (2010). Políticas Públicas de Juventud en América Latina. Debates SHS.

UNIVERSIDAD SURCOLOMBIANA Y MUNICIPIO DE NEIVA. (24 de Mayo de 2015). Convenio Interadministrativo. Actualización de la política pública de juventud. Neiva, Huila, Colombia: ALCALDÍA DE NEIVA.

Vargas Velásquez, A. (1998). Notas sobre los conceptos de sistema y régimen político. Estudios Políticos, 157-180.

Vargas-Velásquez, A. (13 de Marzo de 2017). Necesidad de una política pública criminal. El Colombiano.

Vidal Correa, F. (2014). La participación política en México: Entendiendo la desigualdad entre hombres y mujeres. Revista Mexicana de Ciencias Políticas y Sociales, 317-356.

Vommaro, P. (2013). Las relaciones entre juventudes y políticas en la América Latina contemporánea: una aproximación desde los movimiento estudiantiles. Revista Sociedad, 19.

Vommaro, P. A. (2013). Balance crítico y perspectivas acerca de los estudios sobre juventudes y participación política en la Argentina (1960-2012). Sudamericana, 40 páginas. 